\title{
Development and performance of Bechtel Nevada's Nine-Frame Camera System
}

Stuart A. Baker,* Melissa J. Griffith, Joshua L. Tybo; Bechtel Nevada

\author{
ABSTRACT
}

Bechtel Nevada, Los Alamos Operations, has developed a high-speed, nine-frame camera system that records a sequence from a changing or dynamic scene. The system incorporates an electrostatic image tube with custom gating and deflection electrodes. The framing tube is shuttered with high-speed gating electronics, yielding frame rates of up to 5 MHz. Dynamic scenes are lens-coupled to the camera, which contains a single photocathode gated on and off to control each exposure time. Deflection plates and drive electronics move the frames to different locations on the framing tube output. A single charge-coupled device (CCD) camera then records the phosphor image of all nine frames. This paper discusses setup techniques to optimize system performance. It examines two alternate philosophies for system configuration and respective performance results. We also present performance metrics for system evaluation, experimental results, and applications to four-frame cameras.

Keywords: high-speed imaging, framing camera, electro-optics, CCD camera, system performance

\section{INTRODUCTION}

Bechtel Nevada designed the nine-frame camera system around an electrostatic framing/streak tube manufactured by IMCO, Ltd. ${ }^{1}$ We have developed high-speed gating electronics to precisely control exposure times, from 50 nanoseconds to 5 microseconds. The first camera design was based on a four-frame imaging format. The fact that certain applications require additional frames to record a more continuous image sequence spurred the development of a nine-frame version of this system. The framing camera has a single line of sight optical imaging system and uses a standard Nikon lens to view a dynamic scene. The scene is projected onto the single photocathode of the image tube. The camera's gating circuit controls exposure time. Deflection electronics position each gated image at a different location on the image tube's large phosphor output screen. A single scientific-grade CCD camera then records the image on the phosphor screen containing all nine frames. This paper discusses camera system performance and methods that optimize image quality in resolution, sensitivity, and distortion. Applications for this type of imaging system include radiographic imaging of explosive events and visible light shadowgraphy.

\section{FRAMING CAMERA CONFIGURATION}

The framing camera system operates with a single 18-mm- diameter photocathode and a large-output phosphor screen. The 72-millimeter-diameter, large-area phosphor screen allows multiple image/frame exposures to be displayed. A fiberoptically coupled CCD camera records the phosphor image. In the nine-frame configuration, the CCD readout camera requires many pixels to provide adequate resolution elements for good image quality over a large field of view (FOV). A typical FOV for the imaging systems is 125 millimeters square in the lens-viewed object plane. To maintain good resolution for the large field of view, we prefer a minimum of 1000 pixels per image frame. To maximize the number of square format CCD pixels used in image recording, the photocathode is masked to a square area. Four-frame camera images are positioned in a $2 \times 2$ matrix on the output phosphor screen; nine-frame images, in a $3 \times 3$ matrix. The masked photocathode area and image-tube magnification are set for the frame images to fill the CCD sensor. The photocathode mask size is set to match the recorded object plane FOV and optical input magnification used.

\subsection{Four-frame system}

Our initial framing camera was designed for four-frame exposures. We wanted to take full advantage of the image tube area both on the input photocathode and the large-output phosphor. The largest mask to fit the 18-mm-diameter photocathode is a $12.7-\mathrm{mm}$ square. Initial camera readouts utilized a one-inch or slightly larger sensor, 1024 or 
1600 pixels square. The configuration required the four frames to be mapped onto the one-inch-square sensor. The image tube may be operated with an image magnification from 1.0 to 2.0 (image size on phosphor over image size on photocathode). To utilize the image tube resolution, the photocathode image is expanded onto the phosphor, covering a large area of it. A 2:1 fiber taper transfers and reduces the image size to fit onto the CCD sensor. The fiber-optic taper allows a large area of the phosphor to be recorded, with the trade-off in system resolution limited by the camera's available pixels.
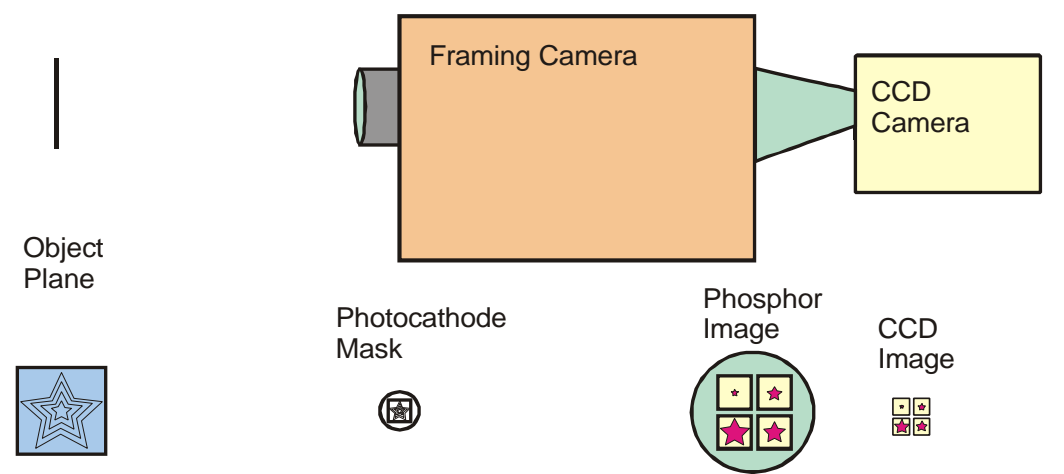

Figure 1. Framing system profile and image layout

\subsection{Nine-frame system}

The initial approach to assembling the nine-frame system used the same technique. Once we developed the requisite electronics to drive the camera in this mode, we knew that recording nine frames with decent resolution would require many pixels. A 4096 x 4096 CCD array provides high-resolution recording of the nine-frame camera image in a $3 \times 3$ frame pattern. This CCD sensor is 61 millimeters square, well-matching it to the phosphor screen area and direct 1:1 fiber-optic coupling of the image.

To maximize the image-tube area used, the optical lens magnification is set to fill a large mask area on the photocathode. The large photocathode area requires the image-tube magnification to be run near its minimum in order to fit into the CCD area. The image tube magnification operating at a minimum improves system sensitivity, or detection efficiency, as the energy on the photocathode is only slightly expanded in size as it is imaged onto the phosphor

\subsubsection{Resolution roll-off}

The first time the complete nine-frame CCD coupling was assembled, we observed a roll-off in resolution in different frames. The image-tube focusing technique used in the past on four-frame cameras proved unsatisfactory for the nineframe version. This initial focus was set by maximizing the resolution of frame number 1 . The camera focus then showed poor resolution in some frames and an average resolution of $1.6 \mathrm{lp} / \mathrm{mm}$ for a 5 -inch FOV over the nine frames. The images included astigmatism and pincushion distortion seen in the 5-inch target pattern.

We attempted to improve the focus by maximizing the resolution in the center frame location. This resulted in high central frame resolution but very poor corner resolution, severe astigmatism, and an average nine-frame resolution of $1.5 \mathrm{lp} / \mathrm{mm}$. The FOV and resolution images provided a high-percentage use of available CCD pixels; however, the astigmatism and pincushion distortions made this configuration undesirable.

\subsubsection{Uniform frame focus}

Using the maximum area on the photocathode for imaging results in severe pincushion distortion of the frame image on the phosphor. Frames positioned near the edge of the phosphor screen result in astigmatism and resolution roll-off. With the major distortions appearing to originate from the extremities of the photocathode and large deflection angles, we 
chose to reduce the image mask size used on the photocathode and enlarge the tube magnification to fill the CCD area. Using a smaller photocathode area allows the image-tube magnification to run near its maximum extreme of 2.0 Expanding the photocathode image onto the phosphor screen reduces energy density or image brightness but provides higher resolution imaging from the photocathode to the phosphor. The resulting images exhibit low distortion and show fine detail in the 5-inch FOV target (Figure 2). The average limiting resolution is $1.9 \mathrm{lp} / \mathrm{mm}$ over the nine-frame's.

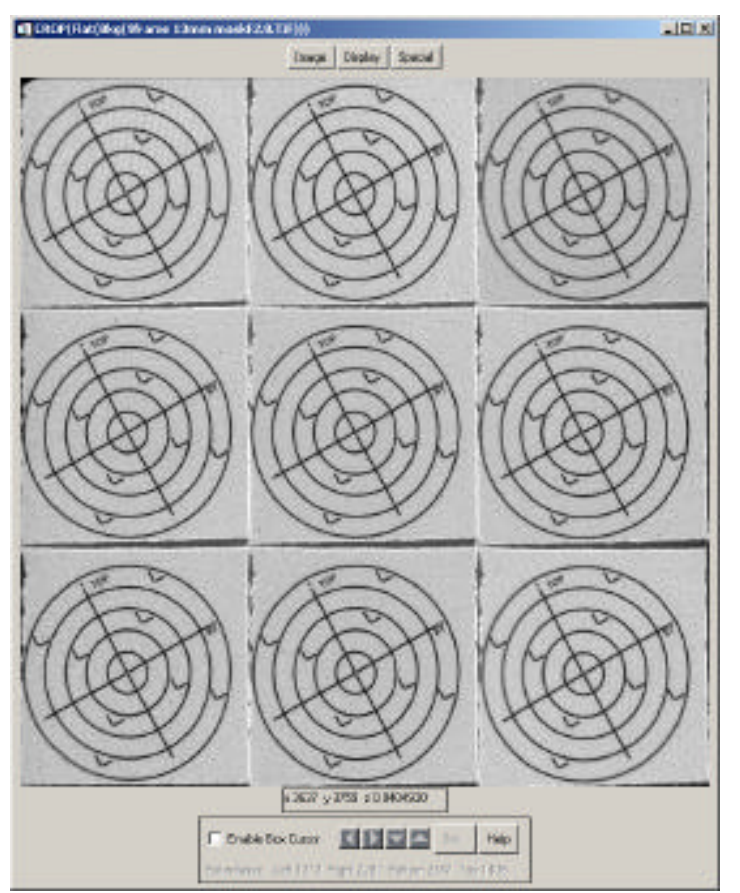

Figure 2. Five-inch FOV target, uniform frame focus, high image-tube magnification

2.2.3. Photocathode mask size

The photocathode mask size has been adjusted to optimize image quality versus mask area, optical magnification must compensate for maintaining FOV. The tradeoff on this adjustment is that as the mask becomes too large, image quality degrades. As the mask gets smaller, system resolution becomes CCD pixel-limited, due to compressing the same FOV into fewer pixels.

In addition to the apparent image distortions with the large mask size, a second factor involves background scatter of electrons inside the image tube. When a large photocathode area is used, background scatter appears as a slight phosphor glow over the entire phosphor screen. The elevated background results in reduced scene contrast, inhibiting detection of fine image detail. Using a smaller photocathode area significantly improves scene contrast.

\section{CAMERA PERFORMANCE}

$\mathrm{BN}$ evaluates camera systems based on their results in a series of performance metrics. Evaluation categories include system sensitivity, dynamic range, and resolution. For electro-optical imaging systems with a digital electronic readout, we measure sensitivity in terms of photocathode quantum efficiency (QE), linearity transfer curve, ${ }^{3}$ and detective quantum efficiency (DQE) ${ }^{4}$ Several of BN's measurement techniques are described in "Evaluating intensified camera systems. ${ }^{, 5}$ System dynamic range may be measured according to linearity transfer curve or photon transfer curves, ${ }^{3}$ both discussed below. Imaging system resolution is based on contrast transfer function (CTF) and modulation transfer function (MTF) measurements. 


\subsection{Camera sensitivity and dynamic range}

Camera system sensitivity calibrations are traditionally measured using the linearity transfer curve shown in Figure 3 This curve indicates the camera system's linear operating range. The curve relates the camera signal level in counts above background to the input energy density incident on the photocathode. This is a straightforward way to calibrate the camera response and allows for basic comparison of various systems. A more useful experimental system calibration is to convert the input energy scale from the photocathode to the object plane being viewed. Camera shot noise is plotted versus input energy to identify a noise equivalent input $(\mathrm{NEI})^{6}$ and signal-to-noise ratio of $2: 1$. The NEI and $\mathrm{S}: \mathrm{N}=2: 1$ input energy levels are indicated on the linearity transfer curve along with digitizer saturation. The NEI indicates the threshold input energy level required to record a detectable signal. Using low-noise readout cameras, it is now possible to record usable data at very low signal levels. Dynamic range measured on the linearity transfer curve indicates the input energy range over which a usable signal may be recorded. Dynamic range is the ratio of input energy for camera saturation to the cameras NEI. It is generally preferred to record signal levels above the $\mathrm{S}: \mathrm{N}=2: 1$ energy level.

Linearity Transfer Curve

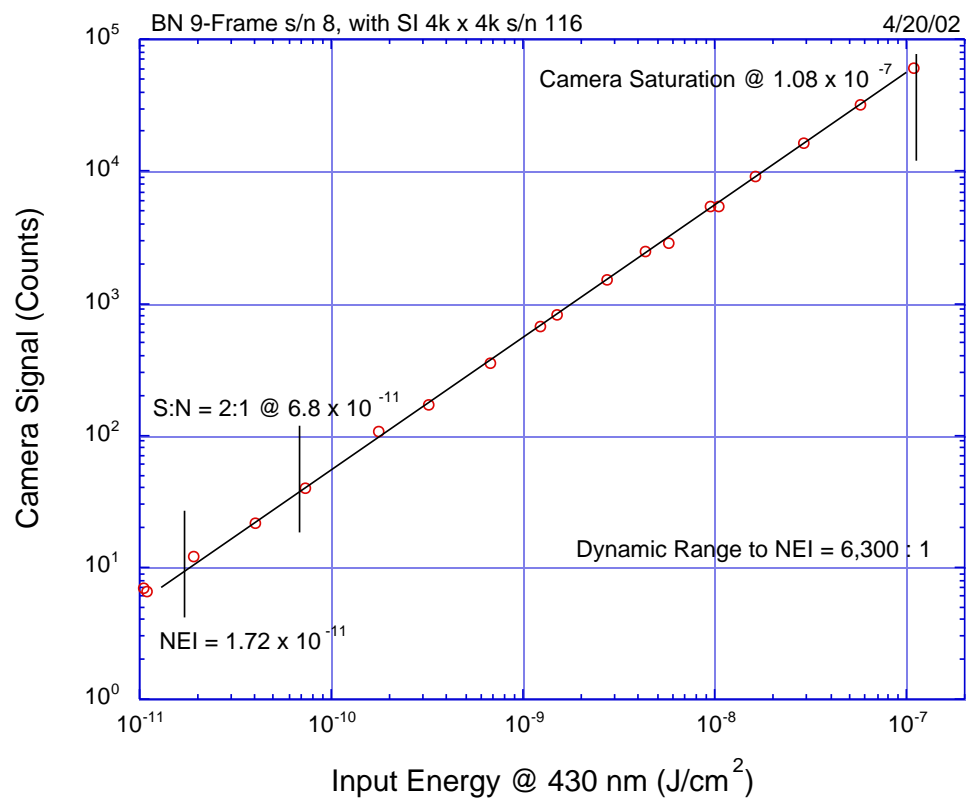

Figure 3. Linearity transfer curve

The camera system's linear region of operation has a slope of 1 . The linearity transfer curve provides an input energy calibration; however, image noise affects the dynamic range measurement. We isolate image noise components using the photon transfer curve, which is traditionally used as a calibration for CCD chips and cameras. ${ }^{3}$ We compute the curve shown in Figure 4 by subtracting two identical exposures and plotting the mean signal level of the exposure versus the resultant noise of the differenced images. By subtracting two identical frame exposures, we remove fixed pattern noise from the image and are left with camera shot noise and CCD read noise. Fixed pattern noise is very evident in the raw framing camera images; they are included by stationary fixtures that are constant in each image frame. Components that contribute to fixed pattern noise signature include the image tube, CCD chip, and fiber-optic components or lenses used as an optical image relay.

The photon transfer curve indicates several features of the imaging system. The plot's linear region has a slope of $1 / 2$ which indicates camera shot noise response with fixed pattern noise removed from the noise measurement. At low signa levels, camera noise begins to level off, as the CCD read noise becomes dominant. When digitizer saturation is reached, the noise drops sharply. Full well capacity, and read noise are computed as effective values for the camera system. The 
effective camera gain constant depends on the image tube characteristics and operating configuration, as well as CCD camera electronics. The linear region of the curve determines the effective camera gain constant, K. The camera gain constant is calculated as:

Camera gain constant, $\mathrm{K}=$ Mean Signal $/\left((\text { Signal Noise })^{2}-(\text { Read Noise })^{2}\right),\left(\mathrm{e}^{-} /\right.$count $)$,

Where Mean Signal is the average signal per pixel above background in counts or digital number, $\mathrm{DN}^{3}$.

Signal Noise is (standard deviation (image 1 - image 2))/sqrt(2).

Read Noise is determined graphically as the minimum noise measured on dark field illumination.

The camera gain constant may also be roughly determined graphically, by extending the linear region slope back to cross camera noise level of 1, as shown in Figure 4. Effective full well is the maximum signal capacity per pixel, and is normally restricted by the saturation or maximum bit count of the digitizer. Full well capacity (counts) and read noise (counts) are converted to electrons (e`) by the camera gain constant, $\mathrm{K}$.

\section{Photon Transfer Curve}

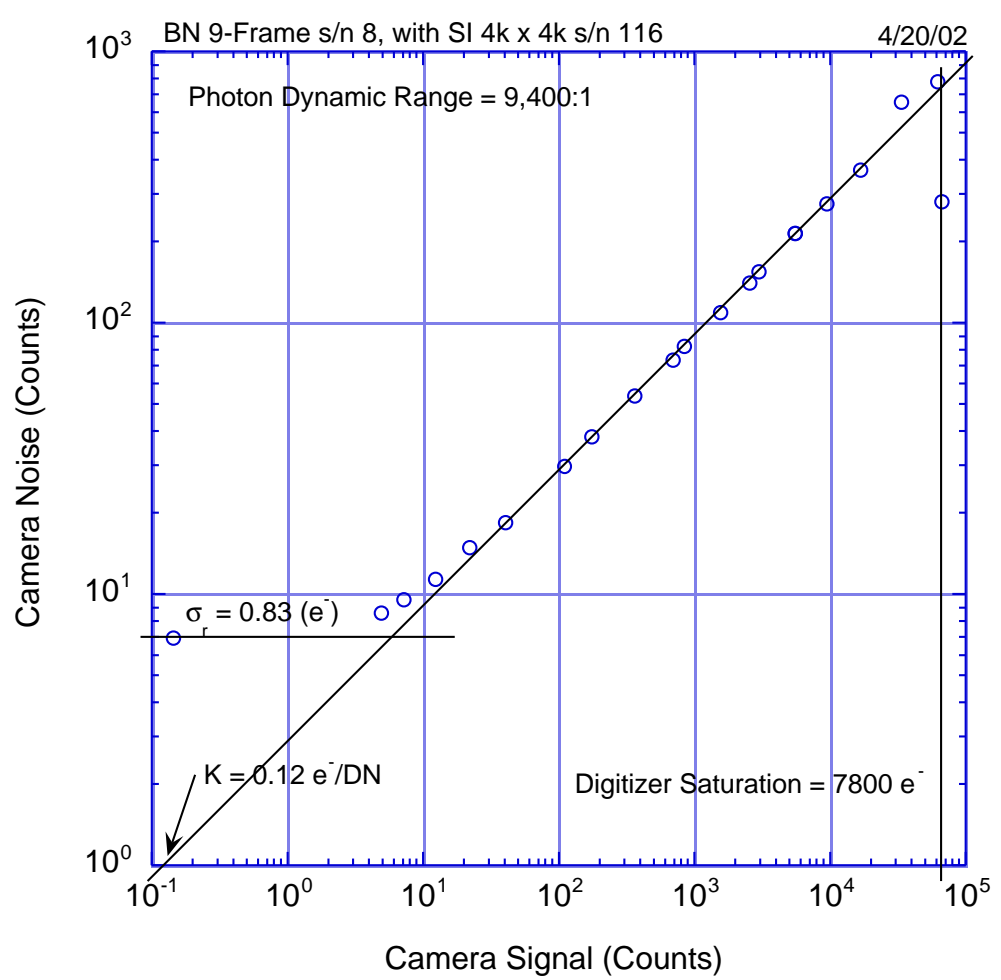

Figure 4. Photon transfer curve

The full well and read noise values indicated on the photon transfer curve are used to calculate the system's maximum dynamic range. This calculation may show a larger dynamic range, since it is computed from the extreme limits of the recording system than the linearity transfer curve. 


\subsection{Camera system resolution}

We initially set up the framing system while viewing the photocathode resolution through the camera system. While this resolution is helpful in camera setup, the element most critical to the system's usefulness is its performance-recording information in the object plane, or scene being recorded. Object plane resolution becomes a system measurement, folding in the photocathode area and the optical lens magnification to image the desired FOV. The modulation transfer function plot shown in Figure 5 is calculated from an edge image in the object plane. ${ }^{5,7,8}$ The MTF plot, the sinusoidal frequency response, is a convenient way to view the resolution of a camera system. Notable points from the plot are the $50 \%$ modulation frequency, identified as the cutoff frequency, $\mathrm{f}_{\mathrm{c}}$, and the limiting resolution spatial frequency, indicated as the modulation approaches $5 \%$, or the maximum detectable resolution.

The MTF plot nicely presents system resolution. However, it is often difficult to focus a camera on the edge target used to generate the MTF, since small changes are barely perceptible without some automated processing. Therefore, standard bar pattern targets are normally used to tweak in focus on an optical system. The contrast transfer function (CTF) measured from the square bar patten target correlates well with the limiting resolution indicated on the MTF plot. The CTF modulation provides a few point checks on the MTF results.

\section{Frame MTF}

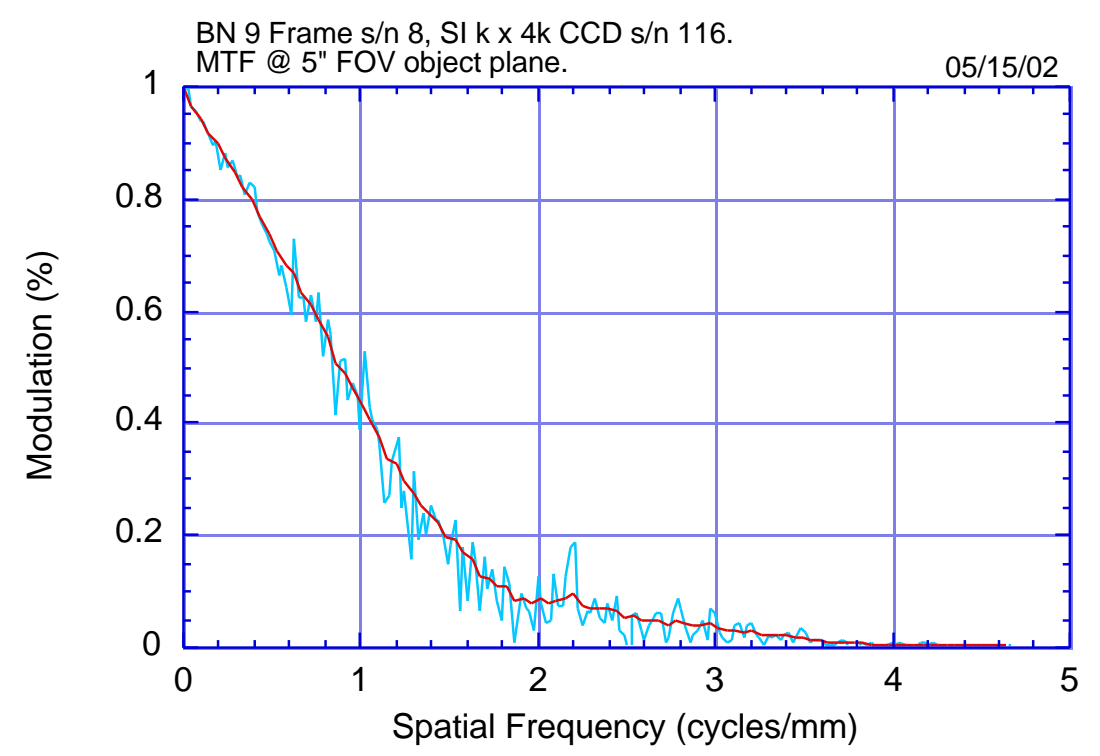

Figure 5. BN Nine-Frame modulation transfer function

One noticeable improvement in resolution from the modified focusing technique discussed earlier is in scene contrast. We have benchmarked scene contrast as the percent modulation, CTF, in a particular $\mathrm{lp} / \mathrm{mm}$ target at the object plane. Scene contrast is measured viewing dark bars on a light background. Scene contrast is affected by light scatter in the image tube and optical imaging system. Scatter creates a loss of sharpness or image blur, referred to as "long-range blur."

In the original framing configuration, long-range blur was readily apparent when viewing an edge target. The blur extending from a light to dark transition extended for many millimeters, or CCD pixels, across the phosphor screen. Long-range blur can obscure image detail near a bright edge. The new focus technique has a much sharper edge response, due to reduced background scatter. 


\section{FOUR-FRAME SYSTEM}

The focusing technique we discovered in setting up the nine-frame has now been applied to a four-frame camera system, where it has lead to similar improvements in imaging resolution. The base resolution, $\mathrm{f}_{\mathrm{c}}$, at the photocathode improved from $3.6 \mathrm{lp} / \mathrm{mm}$ to $10.6 \mathrm{lp} / \mathrm{mm}$ at the center of the photocathode. For all four frames, we tested the four-frame system with a $1024 \times 1024$ pixel CCD array (approximately $500 \times 500$ pixels per frame). As a result, the minimum detectable resolution was pixel-limited. The framing tube images would be better resolved with $1000 \mathrm{x} 1000$ pixels per frame. The framing camera modified focus involved adjusting the tube magnification of the photocathode phosphor screen projection from 0.88 to 2.02 . Operating the framing tube at the high magnification of 2.02 required reducing the photocathode area used so that the four frame images fit onto the CCD chip. We reduced the photocathode mask from 12 millimeters square to 5.6 millimeters square. To compare performance of the two camera configurations, we measured results of viewing the same 5-inch $(127-\mathrm{mm})$ FOV with the available photocathode area. The original configuration, using a $12 \times 12$ millimeter photocathode area, required an optical magnification of 10.6. The new $5.6 \times 5.6$ millimeter photocathode area required an optical magnification of 22.7 to view the $127 \times 127$ millimeter object plane.

The results of this projected camera resolution appear in the 4-frame MTF graph (Figure 6). The four-frame MTF graph shows three plots. These plots are identified by the effective pixel size at photocathode due to the image tube magnification. The 11.9-micron pixel at the photocathode indicates the high tube magnification of 2.02, while the 27.3-micron pixel indicates the low tube magnification. The two plots on the 27.3-micron pixel, low tube magnification setting indicate the resolution roll-off toward the outer edge of the frames due to severe image distortion and long-range blur in this configuration. Long-range blur is apparent in a profile of a sharp edge image (light to dark transition in the image) if the edge profile appears rounded with tailing extending many pixels from the edge.

Long-range blur and distortion appear to be more of a problem in the framing tube when areas near the edge of the photocathode are used. Using the photocathode edge area seems to create excessive photoelectron scatter inside the image tube. This scatter causes long-range blur and a slight phosphor glow over the entire phosphor area, raising the background level and reducing scene contrast. Using the full photocathode area also creates distortions introduced by the high field effects near the edge of electro-optical focusing components in the image tube.

\section{Frame MTF}

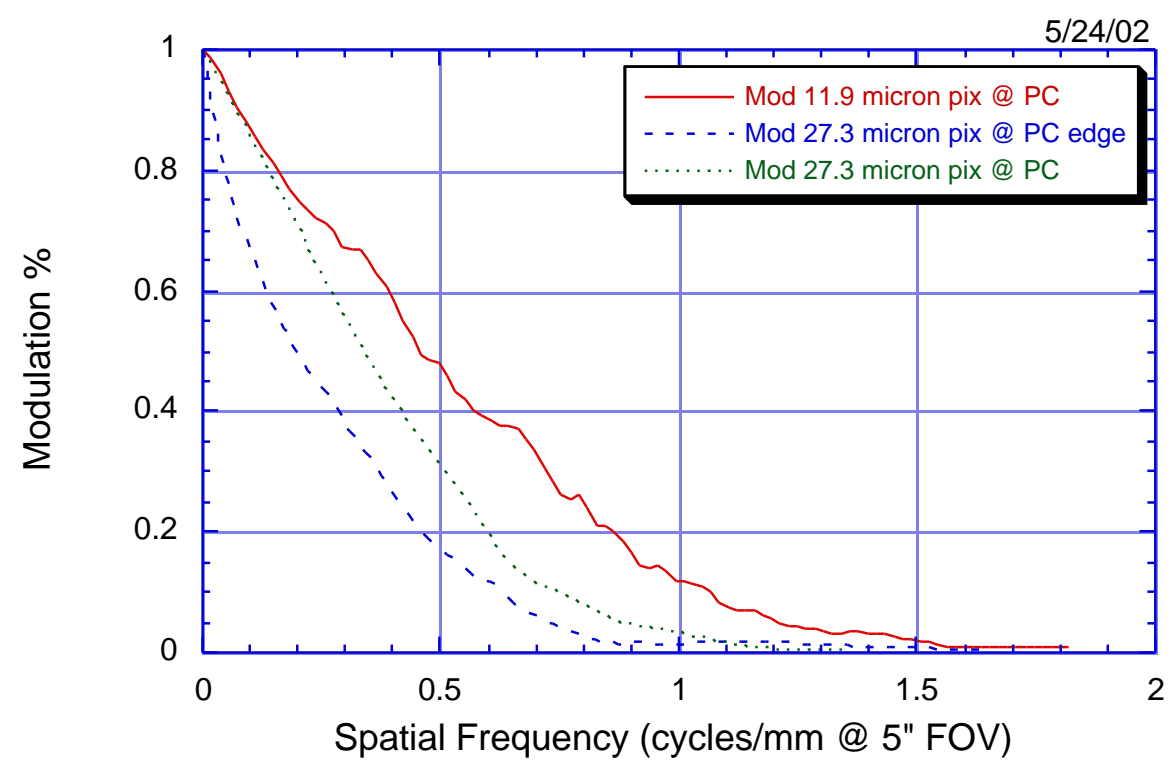

Figure 6. Four-Frame MTF 
The 11.9-micron pixel at the photocathode in the high tube magnification setting shows improved resolution

performance for the 5-inch FOV. This configuration also results in low image distortion and very little long-range blur. The resolution is pixel-limited with the $500 \times 500$ pixels per frame. The nine frame camera, which is set up for approximately 1200 x 1200 pixels per frame, exhibits higher resolution, as shown in Figure 5.

\section{DYNAMIC IMAGE SEQUENCE}

The original BN 9-frame camera system was used to record a dynamic shot sequence in the proton radiography (P-Rad) system at Los Alamos National Laboratory (LANL) in December 2001. The setup configuration of the 9-frame camera was then fine tuned to optimize image quality by matching the object field of view to the image tube area used. Image quality is based on resolution, distortion, sensitivity and signal to noise. The dynamic 9-frame image sequence shown in figure 9 , is an aluminum flyer plate experiment provided by David Holtkamp of LANL. The aluminum plate is shock accelerated by high explosive to study material properties. The aluminum flyer plate radiograph was recorded on June 6 , 2002 at the LANL/P-Rad facility. The image sequence starts with frame one in the lower left corner and progresses counter clockwise around the 3 by 3 frame pattern with frame nine positioned in the center frame location.

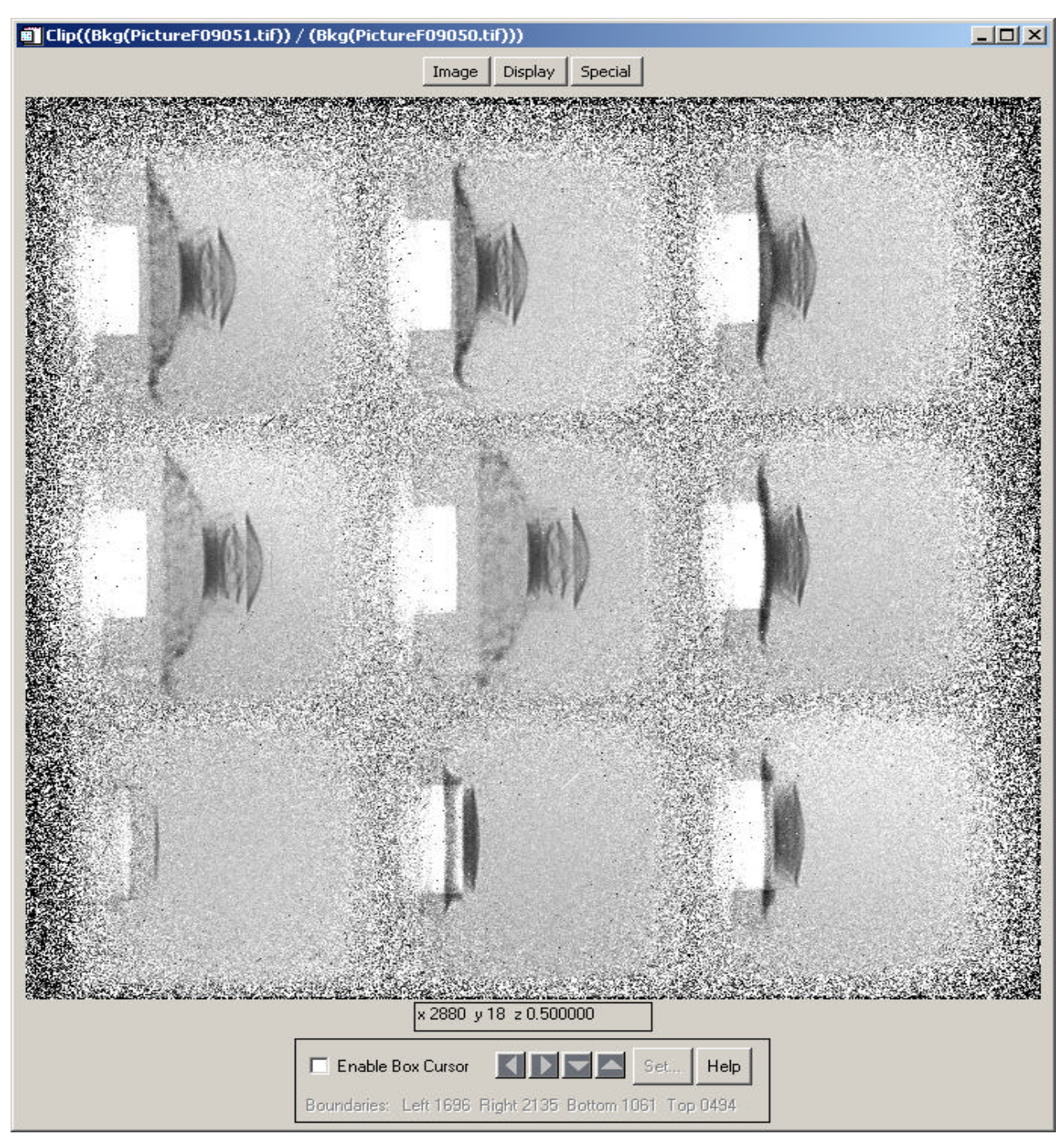

Figure 7. Dynamic image 


\section{CONCLUSION}

The BN nine-frame imaging system has proven to be a useful high-speed imaging diagnostic. Special features that make this system attractive are the single optical axis and photocathode, which provide efficient signal coupling. Another special feature is the high pixel count CCD camera used to record all nine frames in a single image. Operating the framing tube at high, 2.0 magnification provides high resolution imaging through the image tube. Spreading out the photocathode image onto the phosphor screen avoids resolution limits due to the phosphor screen, and allows the tube's electron optics to provide the best imaging resolution of the photocathode. Utilizing the central region of the photocathode greatly reduces electro-optical imaging distortions. The BN nine-frame camera system provides simple high frame-rate-triggering capabilities. Its electronics and mechanical design provide a rugged and serviceable package.

The camera input may be configured for simple lens imaging or fiber-optic bundle image input. Image intensifiers are also an optional input configuration for extremely low-light-level imaging. All readout cameras are fiber-optically coupled to the framing tube using a swivel mount CCD bracket that allows flush coupling of the flat fiber-optic faceplate imaging surfaces. CCD camera to recording computer interface is accomplished with a fiber-optic communication link, to allow the camera to operate in harsh radiation or explosive environments while the recording computer remains in a safe location.

\section{ACKNOWLEDGMENTS}

J. Rohrer, electronics design; Paul Flores, mechanical package design and mounts; Nick King, technical guidance an motivation; Susan D. Gardner, Richard T. Thompson Jr., and Wil Wewis, BN programmatic support. Diagnostic development and experimental data support from Nick King, Chris Morris, Frank Merrill, Gary Hogan, Mary Hockaday, Alexander Saunders, and other LANL/P-Rad collaborators. Experimental shot package provided by David Holtkamp, Kathy Prestridge, and Paul Righltey. This work was supported by the U. S. Department of Energy, National Nuclear Security Administration Nevada Operations Office, under contract No. DE-AC08-96NV11718.

\section{REFERENCES}

1. Imco, Ltd., Mark Riches, image tube design and fabrication.

2. S. A. Baker, S. D. Gardner, M. L. Rogers, F. Sanders, T. W. Tunnell, "ImageTool," Hi-Speed Imaging and Sequence Analysis III, SPIE Vol. 4308, 2001, pp. 1-9.

3. James R. Janesick, Scientific Charge-Coupled Devices. SPIE Press, PO Box 10, Billingham, WA 98227-0010, 2001, pp. 95-119.

4. J. C. Dainty, and R. Shaw, Image Science, Academic Press, Inc. (London) LTD., 24/28 Oval Road, London NW 1974, pp.152-156.

5. S. A. Baker, S. D. Gardner, M. L. Rogers, F. Sanders, "Evaluating intensified camera systems," in Image Intensifier and Applications II, SPIE Vol. 4128 (2000), pp. 99-109.

6. G. C. Holst, CCD Arrays, Cameras, and Displays, SPIE Optical Engineering Press, PO Box 10, Billingham, WA 98227, 1995, pp. 192-193.

7. S. A. Baker, N. S. P. King, S. S. Lutz, D. V. Morgan, T. Schaefer, M. D. Wilke, "Performance of image intensifiers in radiographic systems," in High-Speed Imaging and Sequence Analysis II, SPIE Vol. 3968 (2000), pp. 39-46. 
8. G. D. Boreman, "MTF in optical and electro-optical systems." SPIE short course, Center for Research \& Education in Optics \& Lasers CREOL at University of Central Florida, 1997, pp 94-96.

\section{DISCLAIMER}

This report was prepared as an account of work sponsored by an agency of the United States Government. Neither the United States Government nor any agency thereof, nor any of their employees, nor any of their contractors, subcontractors or their employees, makes any warranty, express or implied, or assumes any legal liability or responsibility for the accuracy, completeness, or any third party's use or the results of such use of any information, apparatus, product, or process disclosed, or represents that its use would not infringe privately owned rights. Reference herein to any specific commercial product, process, or service by trade name, trademark, manufacturer, or otherwise, does not necessarily constitute or imply its endorsement, recommendation, or favoring by the United States Government or any agency thereof or its contractors or subcontractors. The views and opinions of authors expressed herein do not necessarily state or reflect those of the United States Government or any agency thereof.

\section{DISTRIBUTION LIST}

U.S. Department of Energy

National Nuclear Security Administration

Nevada Operations Office

P.O. Box 98518

Las Vegas, NV 89193-8518
U.S. Department of Energy Office of Scientific and Technical Information

P.O. Box 62

Oak Ridge, TN 3783

(electronic copy)
DOE/NNSA

Public Reading Facility

P.O. Box 98521

Las Vegas, NV,

89193-8518 